\title{
ADDITIVE COHOMOLOGY OPERATIONS
}

\author{
JEANNE DUFLOT
}

\begin{abstract}
The bigraded group $\left\{H_{i}\left(\Sigma_{n}, \mathbf{Z} / p\right)\right\}$ becomes a Hopf algebra, if multiplication is induced by restriction, and comultiplication is induced by transfer. Using Steenrod's method of considering elements of this bigraded group as mod- $p$ cohomology operations, the primitives of this Hopf algebra correspond to additive cohomology operations. In this paper we use the results known about the homology and cohomology of the symmetric groups and the operations they induce in mod - $p$ cohomology to write down two (additive) bases of the bigraded vector space of primitives of the above Hopf algebra.
\end{abstract}

\section{INTRODUCTION}

Let $\Sigma_{n}$ denote the symmetric group on $n$ letters. There is a graded Hopf algebra $R_{*}=\bigoplus_{n \geq 0} R_{\mathrm{C}}\left(\Sigma_{n}\right)$ where $R_{\mathrm{C}}\left(\Sigma_{n}\right)$ is the complex representation ring of the symmetric group $\Sigma_{n}$. The multiplication is given by induction of representations, the comultiplication by restriction of representations. Let $R_{*}^{\text {dual }}$ denote the graded Z-dual of $R_{*}$; Atiyah [1] shows how $R_{*}^{\text {dual }}$ embeds in the set of natural transformations of $K^{0}$ (considered as a set-valued functor) and proves directly that the primitives in $R_{*}^{\text {dual }}$ give additive operations on $K^{0}-$ the Adams operations. Moreover, one knows exactly the primitives of $R_{*}$, and therefore also of $R_{*}^{\text {dual }}$ since $R_{*}$ is a self-dual Hopf algebra (see, e.g., [8]).

Now, let $p$ be a fixed prime number. Consider the bigraded group (as in [3]) $H_{* *}=\left\{H^{i}\left(\Sigma_{j}\right)\right\}_{i \geq 0, j \geq 0}$. (All homology and cohomology groups, unless otherwise denoted, have coefficients in $\mathbf{Z} / p$.) In [3], it is pointed out that

(a) $H_{* *}$ is a bigraded Hopf algebra with multiplication induced by transfer; specifically by the transfer maps induced by the (standard) inclusions $\Sigma_{l} \times$ $\Sigma_{m} \rightarrow \Sigma_{l+m}$. Comultiplication is given by restriction maps induced by the above inclusions.

(b) The bigraded dual of $H_{* *}$ is isomorphic as a Hopf algebra to $\widehat{H}_{* *}=$ $\left\{H_{i}\left(\Sigma_{j}\right)\right\}_{i \geq 0, j \geq 0}$, where multiplication is induced by restriction, and comultiplication is induced by transfer.

(c) Using Steenrod's method [21] of considering elements of $H_{*}\left(\Sigma_{n}\right)$ as mod- $p$ cohomology operations, the primitives of $\widehat{H}_{* *}$ (with respect to the transfer comultiplication) correspond to additive cohomology operations. (See

Received by the editors May 1, 1988 .

1980 Mathematics Subject Classification (1985 Revision). Primary 55S05; Secondary 20J06. 
McClure [13] for a much more general proof of this fact.) More precisely, a primitive $\alpha \in H_{i}\left(\Sigma_{n}\right)$ corresponds to a sequence of additive cohomology operations $\hat{\alpha}_{q}: H^{q} \rightarrow H^{n q-i}$. (Here, for simplicity, we assume that $q$ is even if $p$ is odd.) From now on, if $\alpha \in H_{i}\left(\Sigma_{n}\right)$ we denote by $\hat{\alpha}$ or $(\alpha)^{\wedge}$ the cohomology operation corresponding to $\alpha$. If we wish to specify $\hat{\alpha}$ as acting only in the cohomology degree $q$, we write $\hat{\alpha}_{q}$.

In this paper we continue the investigation of [3] by using the results known about the homology and cohomology of the symmetric groups and the operations they induce in mod $-p$ cohomology to write down two (additive) bases of the bigraded vector space of primitives (with respect to the transfer comutiplication) of $\widehat{H}_{* *}$.

We also investigate the wreath product on $\widehat{H}_{* *}$ (which corresponds to composition of cohomology operations).

\section{TWO BASES FOR THE PRIMITIVES IN $\widehat{H}_{* *}$}

Let $P_{* *}$ and $Q_{* *}$ (resp. $\widehat{P}_{* *}$ and $\widehat{Q}_{* *}$ ) denote the primitives and indecomposables of the Hopf algebra $H_{* *}$ (resp. $\widehat{H}_{* *}$ ). Let $V^{n}$ be a vector space of dimension $n$ over $\mathbf{Z} / p$; fix a one-to-one correspondence between $V^{n}$ and $\left\{1,2, \ldots, p^{n}\right\}$. Using this correspondence, the set of translations $E_{n}$ of $V^{n}$ is embedded in $\Sigma_{p^{n}}$; say $i: E_{n} \rightarrow \Sigma_{p^{n}}$ is the embedding. Of course, $E_{n}$ is an elementary abelian $p$-subgroup of $\Sigma_{p^{n}}$ of rank $n$. Let $j: \Sigma_{p^{n-1}} \times \cdots \times \Sigma_{p^{n-1}} \rightarrow \Sigma_{p^{n}}$ be the "usual" embedding.

We will make use of the following lemmas.

Lemma 1 [3]. $Q_{*, N}=P_{*, N}=\widehat{Q}_{*, N}=\widehat{P}_{*, N}=0$ if $N$ is not a power of $p$.

Let $\alpha: G \rightarrow H$ be an inclusion of groups; we make the following table of notation

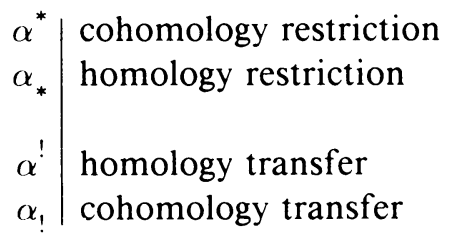

Lemma 2. (a) $Q_{*, p^{n}}=H^{*}\left(\Sigma_{p^{n}}\right) / \operatorname{im} j_{!}$, (b) $\widehat{P}_{*, p^{n}}=\operatorname{ker} j^{!}$.

Proof. Statement (a) is Corollary 7 of [3]. Statement (b) follows from (a) by duality; or can be proved directly using the same methods as for (a).

Lemma 3. (a) $\operatorname{im} j_{!} \subseteq \operatorname{ker} i^{*}$, (b) $\operatorname{im} i_{*} \subseteq \operatorname{ker} j^{!}$.

Proof. (a) is a theorem of Steenrod's; see, e.g., [11 or 16].

(b) follows by duality from (a).

We will also need the following details from calculations of Nakaoka [19] and Cartan [2]. We adopt the notation of the above-cited works of Cartan and Nakaoka. 
Let $\mathbf{Z}^{+}=\{n \in \mathbf{Z} \mid n \geq 0\}$. Let $q$ be a fixed positive even integer. Let $\mathscr{I}_{p}=\left(a_{1}, a_{2}, \ldots, a_{j}, \ldots\right) \in \bigcup_{n \geq 1}\left\{\left(\mathbf{Z}^{+}\right)^{n} \mid \exists i \geq 1\right.$ such that $a_{i} \neq 0, a_{j} \geq p a_{j+1}$ for each $j \geq 1 ; a_{j} \equiv 0$ or $\left.1 \bmod 2(p-1)\right\}$. If $(\quad)$ denotes the empty sequence of integers, let $\mathscr{I}_{p}^{*}=\mathscr{I}_{p} \cup\{(\quad)\}$. If $I=\left(a_{1}, a_{2}, \ldots\right) \in \mathscr{I}_{p}$ define $d(I)=\sum_{i=1}^{\infty} a_{i}$ (the degree of $\left.I\right) ; e(I)=\left[p a_{1} /(p-1)\right]-d(I)$ (the excess of $I) ; l(I)=\max \left\{j \mid a_{j} \neq 0\right\}$ (the length of $I$ ); and $r(I)=p^{l(I)}$ (the rank of $I$ ). Extend these functions to $\mathscr{F}_{p}^{*}$ by defining $d((\quad))=e((\quad))=l((\quad))=0$; so that $r(())=1$. As in Nakaoka [19], define $\Omega(p, q)=\left\{I \in \mathscr{I}_{p}^{*} \mid e(I)<q\right.$; and if $l(I)=j$ then $a_{j}>1$ when $\left.I=\left(a_{1}, \ldots, a_{j}, 0, \ldots\right)\right\}$. Define $U(p, q)$ to be the $\mathrm{Z} / p$-algebra generated by all elements $I \in \Omega(p, q)$ subject to the relations $I J=(-1)^{d(I) d(J)} J I$ for $I, J \in \Omega(p, q)$. The algebra $U(p, q)$ is of course generated as a vector space by monomials $I_{1}^{\alpha_{1}} \cdots I_{k}^{\alpha_{k}}$; one can extend the definition of rank to the set of nonzero monomials so that $r\left(I_{1}^{\alpha_{1}} \cdots I_{k}^{\alpha_{k}}\right)=$ $\sum_{j=1}^{k} \alpha_{j} r\left(I_{j}\right)$ for each nonzero monomial $I_{1}^{\alpha_{1}} \cdots I_{k}^{\alpha_{k}}$. One can also define the $q$-degree of a nonzero monomial as

$$
q-\operatorname{deg}\left(I_{1}^{\alpha_{1}} \cdots I_{k}^{\alpha_{k}}\right)=\sum_{j=1}^{k} \alpha_{j}\left(q+d\left(I_{j}\right)\right) .
$$

(Of course, $r(0)=0=q-\operatorname{deg}(0)$.) We then define $U(p, q)^{d}$ to be the subspace of $U(p, q)$ generated by all monomials of $q$-degree $d$, and $U(p, q)_{r}^{d}$ to be the subspace of $U(p, q)^{d}$ generated by all monomials of $q$-degree $d$ and rank $r$. Then $U(p, q)=\bigoplus_{d>0, r \geq 0} U(p, q)_{r}^{d}$ becomes a bigraded algebra over $\mathbf{Z} / p$.

As in Cartan [2], for each $I=\left(a_{1}, a_{2}, \ldots\right) \in \mathscr{J}_{p}$, define the natural transformation $S t^{I}: H^{n}(-, \mathbf{Z} / p) \rightarrow H^{n+d(I)}(-, \mathbf{Z} / p)$ by $S t^{I}=S t^{a_{1}} \circ S t^{a_{2}} \circ \cdots$ where

$$
S t^{a}= \begin{cases}S q^{a}, & p=2, \\ \beta^{\varepsilon} \mathscr{P}^{s}, & p>2 \text { and } a=2 S(p-1)+\varepsilon .\end{cases}
$$

For $I=(\quad)$, define $S t^{I}=$ identity.

Now, we consider the symmetric products $S P^{m} S^{q}$ of the $q$-sphere for $0 \leq$ $m \leq \infty$; let $i_{m, n}: S P^{m} S^{q} \rightarrow S P^{n} S^{q}$ for $0 \leq m \leq n \leq \infty$ denote the usual inclusions. Using the Dold-Thom theorem, we recognize $S P^{\infty} S^{q}$ as an EilenbergMac Lane space $K(\mathbf{Z}, q)$. Let $l_{m}$ be a generator for $H^{q}\left(S P^{m} S^{q}\right) \cong \mathbf{Z} / p$ for $0 \leq m \leq \infty$; assume that these generators are chosen so that $i_{m, n}^{*}\left(l_{n}\right)=l_{m}$ for $0 \leq m \leq n \leq \infty$.

Nakaoka [19] defined homomorphisms of $\mathbf{Z} / p$-algebras

$$
T_{m}: U(p, q) \rightarrow H^{*}\left(S P^{m} S^{q}\right) \text { for } 0 \leq m \leq \infty
$$

by $T_{m}(I)=S t^{I}\left(l_{m}\right)$; notice that $T_{m}\left(U(p, q)^{d}\right) \subseteq H^{d}\left(S P^{m} S^{q}\right)$. Define the vector space homomorphisms

$$
\Phi_{\alpha, m}: H_{r}\left(\Sigma_{m}\right) \rightarrow H^{m q-\alpha}\left(S P^{\infty} S^{q}\right)=H^{m q-\alpha}(K(\mathbf{Z}, q))=H^{m q-\alpha}(\mathbf{Z}, q, \mathbf{Z} / p)
$$


and

$$
\kappa_{\alpha, m}: H_{\alpha}\left(\Sigma_{m}\right) \rightarrow H^{m q-\alpha}\left(S P^{m} S^{q}\right) \quad(\text { for } 0 \leq m<\infty)
$$

by $\Phi_{\alpha, m}(c)=\hat{c}_{q}\left(l_{\infty}\right)$ and $\kappa_{\alpha, m}(c)=\hat{c}_{q}\left(l_{m}\right)$.

Theorem 4 (Nakaoka [19], Cartan [2], Steenrod). If $q>\alpha+1$, there exist vector spaces $V_{\alpha, m}$ and vector space homomorphisms $\xi_{\alpha, m}: V_{\alpha, m} \rightarrow H_{\alpha}\left(\Sigma_{m}\right)$, $\chi_{\alpha, m}: V_{\alpha, m} \rightarrow U(p, q)_{m}^{m q-\alpha} \quad(m<0$; see Nakaoka [19] for a precise definition of $V_{\alpha, m}, \xi_{\alpha, m}$ and $\left.\chi_{\alpha, m}\right)$ such that

(a) the diagrams below commute

$$
\begin{aligned}
& V_{\alpha, m} \stackrel{\xi_{n, m}}{\longrightarrow} \quad H_{\alpha \alpha}\left(\Sigma_{m}\right) \\
& \chi_{n, m} \downarrow \quad \kappa_{n, m} \downarrow \quad(m<\infty), \\
& U(p, q)_{m}^{m q-\alpha} \underset{\widetilde{T}_{m}=T_{m} \mid U(p, q)_{m}^{m q-\alpha}}{\longrightarrow} H^{m q-\alpha}\left(S P^{m} S^{q}\right) \\
& V_{\alpha, m} \stackrel{\xi_{\alpha, m}}{\longrightarrow} H_{\alpha}\left(\Sigma_{m}\right) \\
& \left.\chi_{n, m, \infty} \downarrow \quad \Phi_{n, m}\right\rfloor \\
& U(p, q)^{m q-\alpha} \underset{T_{\infty}}{\longrightarrow} H^{m q-\alpha}(\mathbf{Z}, q, \mathbf{Z} / p)
\end{aligned}
$$

where $\chi_{\alpha, m, \infty}=\lambda \circ \chi_{\alpha, m}$ with $\lambda: U(p, q)_{m}^{m q-\alpha} \hookrightarrow U(p, q)^{m q-\alpha}$.

(b) $\xi_{\alpha, m}, \kappa_{\alpha, m}, \chi_{\alpha, m}$ and $T_{m}$ are isomorphisms for $m<\infty$. (For $\xi_{\alpha, m}, \chi_{\alpha, m}$ and $T_{m}$ this is due to Nakaoka [19]; for $\kappa_{\alpha, m}$, this is due to Steenrod, see [19].)

(c) $T_{\infty}$ is an isomorphism (Cartan [2], Nakaoka [19]).

(d) $\Phi_{c, m}$ is injective (Steenrod; see [19]).

Proof. Nakaoka [19] proves commutativity of the first diagram in (a) directly, but the same proof works for the second diagram in (a). For (b), (c), (d) refer to the references cited in the theorem.

Corollary 5. $\Phi_{\alpha, m}=T_{\infty} \circ \lambda \circ \widetilde{T}_{m}^{-1} \circ \kappa_{\alpha, m}$; where $\lambda: U(p, q)_{m}^{m q-\alpha} \hookrightarrow U(p, q)^{m q-\alpha}$ is as in Theorem 4 .

Let $A^{d}(\mathbf{Z}, q, \mathbf{Z} / p)$ be the subspace of $H^{d}(\mathbf{Z}, q, \mathbf{Z} / p)$ consisting of the additive natural transformations $H^{q}(-, \mathbf{Z}) \rightarrow H^{d}(-, \mathbf{Z} / p)$. From now on, fix $\alpha$; assume $q$ is an even integer such that $q>\alpha+1$; let $m=p^{n}$ for some $n \geq 0$.

\section{Corollary 6.}

$$
\Phi_{\alpha, p^{n}}\left(\widehat{P}_{\alpha, p^{n}}\right) \subseteq T_{\infty} \lambda\left(U(p, q)_{p^{n}}^{p^{n} q-\alpha}\right) \cap A^{p^{n} q-\alpha}(\mathbf{Z}, q, \mathbf{Z} / p) .
$$

Proof. Corollary 5 shows that

$$
\Phi_{\alpha, p^{n}}\left(\widehat{P}_{\alpha, p^{n}}\right) \subseteq T_{\infty} \lambda\left(U(p, q)_{p^{n}}^{p^{n} q-\alpha}\right) .
$$

Let $A^{p^{n} q-a}(\mathbf{Z}, q, \mathbf{Z} / p)$ be the subspace of

$$
H^{p^{n} q-c \chi}(\mathbf{Z} / p, q, \mathbf{Z} / p)=H^{p^{n} q-\alpha \chi}(K(\mathbf{Z} / p, q), \mathbf{Z} / p)
$$


consisting of the additive natural transformations

$$
H^{q}(-, \mathbf{Z} / p) \rightarrow H^{p^{n} q-\alpha}(-, \mathbf{Z} / p) .
$$

Let $\widetilde{\Phi}_{\alpha, p^{n}}: H_{\alpha}\left(\Sigma_{p^{n}}\right) \rightarrow H^{p^{n} q-\alpha}(\mathbf{Z} / p, q, \mathbf{Z} / p)$ given by $c \mapsto \hat{c}_{q}(l)$ where $l$ is a suitably chosen generator of $H^{q}(\mathbf{Z} / p, q, \mathbf{Z} / p) \cong \mathbf{Z} / p$. Then there is a commutative diagram

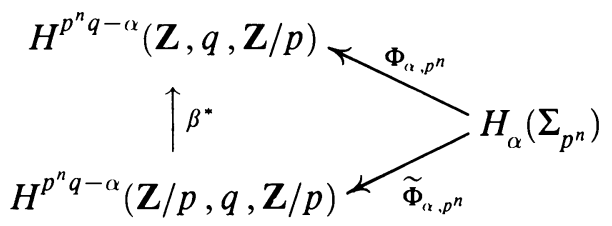

(where $\beta: K(\mathbf{Z}, q) \rightarrow K(\mathbf{Z} / p, q)$ is the map defined by the homomorphism $\mathbf{Z} \rightarrow \mathbf{Z} / p)$ such that $\beta^{*}\left(A^{p^{n} q-\alpha}(\mathbf{Z} / p, q, \mathbf{Z} / p)\right) \subseteq A^{p^{n} q-\alpha}(\mathbf{Z}, q, \mathbf{Z} / p)$. To see this, choose $l$ so that $\beta^{*}(l)=l_{\infty}$. The diagram then commutes because $\hat{c}_{q}$ is natural. Also, $A^{*}(-, q, \mathbf{Z} / p)$ defines a functor on the category of abelian groups (Cartan [2]) so $\beta^{*}\left(A^{p^{n} q-\alpha}(\mathbf{Z} / p, q, \mathbf{Z} / p)\right) \subseteq A^{p^{n} q-\alpha}(\mathbf{Z}, q, \mathbf{Z} / p)$. Now, by [3 or 13], $\widetilde{\boldsymbol{\Phi}}_{\alpha, p^{n}}\left(\widehat{\boldsymbol{P}}_{\alpha, p^{n}}\right) \subseteq A^{p^{n} q-\alpha}(\mathbf{Z} / p, q, \mathbf{Z} / p)$; Corollary 6 follows.

Define ${ }_{p^{n}} H^{p^{n} q-\alpha}(\mathbf{Z}, q)=T_{\infty} \lambda\left(U(p, q)_{p^{n}}^{p^{n} q-\alpha}\right)$.

Corollary 7. For $n \geq 0$, and $\alpha \geq 0$,

(a)

$$
\begin{aligned}
& \operatorname{dim}\left({ }_{p^{n}} H^{p^{n} q-\alpha}(\mathbf{Z}, q) \cap A^{p^{n} q-\alpha}(\mathbf{Z}, q, \mathbf{Z} / p)\right) \\
& \geq \operatorname{dim}\left(\widehat{P}_{\alpha, p^{n}}\right)=\operatorname{dim}\left(\operatorname{ker} j^{\prime}\right)_{\alpha} \geq \operatorname{dim}\left(\operatorname{im} i_{*}\right)_{\alpha},
\end{aligned}
$$

(b)

$$
\begin{aligned}
& \operatorname{dim}\left({ }_{p^{n}} H^{p^{n} q-\alpha}(\mathbf{Z}, q) \cap A^{p^{n} q-\alpha}(\mathbf{Z}, q, \mathbf{Z} / p)\right) \\
& \quad \geq \operatorname{dim}\left(\widehat{P}_{\alpha, p^{n}}\right)=\operatorname{dim}\left(Q_{\alpha, p^{n}}\right)=\operatorname{dim}\left(\operatorname{cok} j_{!}\right)_{\alpha} \geq \operatorname{dim}\left(\operatorname{im} i^{*}\right)_{\alpha} .
\end{aligned}
$$

Proof. The first inequalities follow from Corollary 6 , the middle equalities from Lemma 2, and the last inequalities from Lemma 3.

In fact, all the numbers in Corollary 7 are equal. It is possible to show this using results in Mann [11] to construct a proof (by counting bases) that $\operatorname{dim}\left(\operatorname{im} j_{!}\right)_{\alpha}=\operatorname{dim}\left(\operatorname{ker} i^{*}\right)_{\alpha}$ for each $\alpha \geq 0$ and $n \geq 0$. However, we will prove (by counting bases).

\section{Theorem 8.}

$$
\operatorname{dim}\left(\operatorname{im} i_{*}\right)_{\alpha}=\operatorname{dim}\left(\operatorname{im} i^{*}\right)_{\alpha}=\operatorname{dim}\left({ }_{p^{n}} H^{p^{n} q-\alpha}(\mathbf{Z}, q) \cap A^{p^{n} q-\alpha}(\mathbf{Z}, q, \mathbf{Z} / p)\right)
$$

for $\alpha \geq 0$ and $n \geq 0$.

Proof. This theorem follows directly from the following Theorems 9 and 10 , Corollary 11, and Lemma 12 (plus duality for the first equality). 
Let $A(p, q, n, \alpha)=\left\{I \in \Omega(p, q) \mid r(I)=p^{n}, q+d(I)=p^{n} q-\alpha\right\}$, if $\alpha \geq 0$ and $n \geq 0$.

Theorem 9 (Cartan $[2, \S 16$, Theorem 1]).

$$
\operatorname{dim}\left({ }_{p^{n}} H^{p^{n} q-\alpha}(\mathbf{Z}, q) \cap A^{p^{n} q-\alpha}(\mathbf{Z}, q, \mathbf{Z} / p)\right)=\# A(p, q, n, \alpha)
$$

for $\alpha \geq 0$ and $n \geq 0$.

In the following theorem and corollary, we use the notation of Mui [16].

Theorem 10 (Mui [16], Mann [11], Milgram-Madsen [10] (for $p=2$ )).

(a) If $p=2$, then there are elements $Q_{n, i} \in H^{2^{n}-2^{i}}\left(E_{n}\right)$ for $0 \leq i \leq n-1$ such that $\operatorname{im} i^{*}=\mathbf{Z} / 2\left[Q_{n, 0}, \ldots, Q_{n, n-1}\right]$. (If $K$ is a field, $K\left[x_{1}, \ldots, x_{n}\right]$ means the (graded) polynomial ring over $K$ on $\left\{x_{1}, \ldots, x_{n}\right\}$.)

(b) If $p$ is odd, then there are elements $Q_{n, i} \in H^{2\left(p^{n}-p^{i}\right)}\left(E_{n}\right)$ for $0 \leq i \leq n-1$; $R_{n, i} \in H^{2\left(p^{n}-p^{i}\right)-1}\left(E_{n}\right)$ for $0 \leq i \leq n-1$ and $R_{n, i, j} \in H^{2\left(p^{n}-p^{i}-p^{j}\right)}\left(E_{n}\right)$ for $0 \leq i<j \leq n-1$ such that

$$
\operatorname{im} i^{*}=\frac{\mathbf{Z} / p\left[Q_{n, i}, R_{n, i}, R_{n, i, j} \mid 0 \leq i<j \leq n-1\right]}{\left(\left\{R_{n, i}^{2}, R_{n, i} R_{n, j}-R_{n, i, j} Q_{n, 0} \mid 0 \leq i<j \leq n-1\right\}\right)} .
$$

(The notation $(S)$ means the ideal generated by the set $S$. The product here is the cup product.)

Corollary 11. Let $\left(\operatorname{im} i^{*}\right)_{\alpha}$ denote the homogeneous elements of degree $\alpha$ in $\operatorname{im} i^{*}$. Then

(a) if $p=2$, a basis (over $\mathbf{Z} / 2)$ of $\left(\operatorname{im} i^{*}\right)_{\alpha}$ is given by

$$
\left\{Q_{n, 0}^{r_{0}} Q_{n, 1}^{r_{1}} \cdots Q_{n, n-1}^{r_{n-1}} \mid \sum_{i=0}^{n-1} r_{i}\left(2^{n}-2^{i}\right)=\alpha \text { and } r_{i} \geq 0 \text { for } 0 \leq i \leq n-1\right\} \text {. }
$$

(b) if $p$ is odd, a basis (over $\mathbf{Z} / p$ ) of $\left(\mathrm{im} i^{*}\right)_{1}$ is given by the union of the following two sets:

$$
\begin{aligned}
& \left\{R_{n, \lambda_{0}} R_{n, \lambda_{1}, \lambda_{2}} \cdots R_{n, \lambda_{2 t} \mid \dot{\lambda}_{21}} Q_{n, 0}^{r_{0}} Q_{n, 1}^{r_{1}} \cdots Q_{n, n-1}^{r_{n-1}} \mid\left\{\lambda_{1}, \ldots, \lambda_{2 i}\right\}\right. \\
& \quad \subseteq\{0,1,2, \ldots, n-1\} \text { and } 0 \leq \lambda_{0}<\lambda_{1}<\cdots<\lambda_{2 i} \leq n-1 ; r_{j} \geq 0 \text { for } \\
& \left.\quad 0 \leq j \leq n-1 ; \sum_{j=1}^{2 i}\left(p^{n}-2 p^{\lambda_{\jmath}}\right)+\left(2\left(p^{n}-p^{\lambda_{0}}\right)-1\right)+\sum_{j=0}^{n-1} 2 r_{j}\left(p^{n}-p^{j}\right)=\alpha\right\}
\end{aligned}
$$


and

$$
\begin{gathered}
\left\{R_{n, \lambda_{1}, \lambda_{2}} \cdots R_{n, \lambda_{2 i-1}, \lambda_{2 i}} Q_{n, 0}^{r_{0}} Q_{n, 1}^{r_{1}} \cdots Q_{n, n-1}^{r_{n-1}} \mid\left\{\lambda_{1}, \ldots, \lambda_{2 i}\right\}\right. \\
\subseteq\{0,1, \ldots, n-1\} \text { and } 0 \leq \lambda_{1}<\cdots<\lambda_{2 i} \leq n-1 ; r_{j} \geq 0 \\
\left.\quad \text { for } 0 \leq j \leq n-1 ; \sum_{j=1}^{2 i}\left(p^{n}-2 p^{\lambda_{j}}\right)+\sum_{j=0}^{n-1} 2 r_{j}\left(p^{n}-p^{j}\right)=\alpha\right\} .
\end{gathered}
$$

Proof. Left as an exercise using Theorem 10.

Let

$$
X(2, n, \alpha)=\left\{\left(r_{0}, r_{1}, \ldots, r_{n-1}\right) \in\left(\mathbf{Z}^{+}\right)^{n} \mid \sum_{i=0}^{n-1} r_{i}\left(2^{n}-2^{i}\right)=\alpha\right\}
$$

and if $p$ is odd,

$$
\begin{aligned}
X_{1}(p, n, \alpha)=\{ & \left(\left\{\lambda_{0}, \lambda_{1}, \ldots, \lambda_{2 i}\right\},\left(r_{0}, \ldots, r_{n-1}\right)\right) \\
\in & \mathscr{P}_{2 i+1}([0, n-1]) \times\left(\mathbf{Z}^{+}\right)^{n} \mid 0 \leq i \leq\left(2^{n-1}-1\right) ; \\
& \left.\sum_{j=1}^{2 i}\left(p^{n}-2 p^{\lambda_{j}}\right)+\left[2\left(p^{n}-p^{\lambda_{0}}\right)-1\right]+\sum_{j=0}^{n-1} 2 r_{j}\left(p^{n}-p^{j}\right)=\alpha\right\}
\end{aligned}
$$

and

$$
\begin{gathered}
X_{2}(p, n, \alpha)=\left\{\left(S,\left(r_{0}, \ldots, r_{n-1}\right)\right) \in \mathscr{P}_{2 i}([0, n-1]) \times\left(\mathbf{Z}^{+}\right)^{n} \mid 0 \leq i \leq 2^{n-1} ;\right. \\
\quad \text { if } 0<i \text { and } S=\left\{\lambda_{1}, \ldots, \lambda_{2 i}\right\}, \\
\text { then } \sum_{j=1}^{2 i}\left(p^{n}-2 p^{\lambda_{j}}\right)+\sum_{j=0}^{n-1} 2 r_{j}\left(p^{n}-p^{j}\right)=\alpha ; \\
\text { if } \left.i=0 \text { then } \sum_{j=0}^{n-1} 2 r_{j}\left(p^{n}-p^{j}\right)=\alpha\right\} .
\end{gathered}
$$

(Here, $[0, n-1]=\{0, \ldots, n-1\} ; \mathscr{P}([0, n-1])$ is the power set of $[0, n-1]$; $\mathscr{P}_{k}([0, n-1])$ is the subset of $\mathscr{P}([0, n-1])$ consisting of sets of order $k$. When we write elements of $\mathscr{P}([0, n-1])$ in the form $\{a, b, \ldots\}$ we will always assume that they are written in increasing order.)

Lemma 12. (Compare with May [12], Madsen [9].)

(a) If $p=2$ there is a one-to-one correspondence

$$
F: A(2, q, n, \alpha) \longleftrightarrow X(2, n, \alpha) .
$$


(b) If $p$ is odd, there is a one-to-one correspondence

$$
F: A(p, q, n, \alpha) \longleftrightarrow X_{1}(p, n, \alpha) \amalg X_{2}(p, n, \alpha) .
$$

Proof. (Recall that $q>\alpha+1$.) (a) $F\left(a_{1}, \ldots, a_{n}\right)=\left(q-a_{1}+a_{2}+\cdots+a_{n}\right.$, $\left.a_{1}-2 a_{2}, \ldots, a_{n}-2 a_{n-1}\right)$.

(b) If $S \in \mathscr{P}([0, n-1])$ let $c(S):[0, n-1] \rightarrow \mathbf{Z}$ be the choice function:

$$
c(S)(k)= \begin{cases}0, & k \notin S, \\ 1, & k \in S .\end{cases}
$$

Define $F$ by

$$
\begin{aligned}
& F\left(a_{1}, a_{2}, \ldots, a_{n}\right)=F\left(2 s_{1}(p-1)+\varepsilon_{1}, \ldots, 2 s_{n}(p-1)+\varepsilon_{n}\right) \\
& =\left\{\begin{array}{rr}
\left(S\left(\varepsilon_{1}, \ldots, \varepsilon_{n}\right),\right. \\
\left.\left(\left[q-e_{1}\left(a_{1}, \ldots, a_{n}\right)\right] / 2, s_{1}-p s_{2}-\varepsilon_{2}, \ldots, s_{n-1}-p s_{n}-\varepsilon_{n}\right)\right) \\
\left(S\left(\varepsilon_{1}, \ldots, \varepsilon_{n}\right),\right. \\
\left.\left(\left[q-e_{1}\left(a_{1}, \ldots, a_{n}\right)-1\right] / 2, s_{1}-p s_{2}-\varepsilon_{2}, \ldots, s_{n-1}-p s_{n}-\varepsilon_{n}\right)\right) \\
\text { if } \# S\left(\varepsilon_{1}, \ldots, \varepsilon_{n}\right) \text { is odd }
\end{array}\right.
\end{aligned}
$$

where

$$
\begin{aligned}
e_{1}\left(a_{1}, \ldots, a_{n}\right) & =e_{1}\left(2 s_{1}(p-1)+\varepsilon_{1}, \ldots, 2 s_{n}(p-1)+\varepsilon_{n}\right) \\
& =2 s_{1} p+2 \varepsilon_{1}-\sum_{i=1}^{n} a_{i}=e\left(a_{1}, \ldots, a_{n}\right)+\varepsilon_{1}
\end{aligned}
$$

and $S\left(\varepsilon_{1}, \ldots, \varepsilon_{n}\right) \subseteq[0, n-1]$ is defined by $c\left(S\left(\varepsilon_{1}, \ldots, \varepsilon_{n}\right)\right)(i)=\varepsilon_{i+1}$.

Therefore, one basis for $\widehat{P}_{c, p^{n}}$ (which we will call the Dickson basis) is given as follows. For any $n \geq 0, H^{\alpha}\left(\Sigma_{p^{n}}\right) \cong\left(\operatorname{ker} i^{*}\right)_{\alpha} \oplus\left(\operatorname{im} i^{*}\right)_{\alpha}$, as vector spaces. If $p=2$, for each $R \in X(2, n, \alpha)$, define $D_{R} \in\left(H^{\alpha}\left(\Sigma_{2^{n}}\right)\right)^{\text {dual }} \cong H_{1 \alpha}\left(\Sigma_{2^{n}}\right)$ by $\left.D_{R}\right|_{\left(\operatorname{ker} i^{*}\right)_{1}} \equiv 0$, and $\left\{\left.D_{R}\right|_{\left(\operatorname{im} i^{*}\right)_{1}} \mid R \in X(2, n, \alpha)\right\}$ is the dual basis to the basis of $\left(\mathrm{im} i^{*}\right)_{\alpha}$ given by Corollary 11. If $p$ is odd, for each $(\Lambda, R) \in$ $X_{1}(p, n, \alpha) \cup X_{2}(p, n, \alpha)$ define $D_{(\Lambda, R)} \in\left(H^{\alpha}\left(\Sigma_{p^{n}}\right)\right)^{\text {dual }}$ by $\left.D_{(\Lambda, R)}\right|_{\left(\operatorname{ker} i^{*}\right)_{1,}} \equiv 0$ and $\left\{\left.D_{(\Lambda, R)}\right|_{\left(\operatorname{im} i^{*}\right)_{a}} \mid(\Lambda, R) \in X_{1}(p, n, \alpha) \cup X_{2}(p, n, \alpha)\right\}$ is the basis dual to the basis of $\left(\operatorname{im} i^{*}\right)_{\alpha}$ given in Corollary 11. Then we have seen that $\left\{D_{R} \mid R \in\right.$ $X(2, n, \alpha)\}$ is a basis for $\widehat{P}_{a, 2^{n}}$ and $\left\{D_{(\Lambda, R)} \mid(\Lambda, R) \in X_{1}(p, n, \alpha) \cup X_{2}(p, n, \alpha)\right\}$ is a basis for $\widehat{P}_{\alpha, p^{n}}$ if $p$ is odd.

To get a second basis for $\widehat{P}_{c, p^{n}}$, we use a theorem of Nakaoka's:

Theorem 13 (Nakaoka [19]). For each $I \in A(p, q, n, \alpha)$ there exists an element $b(I)$ in $H_{\alpha \alpha}\left(\Sigma_{p^{n}}\right)$; moreover $\{b(I) \mid I \in A(p, q, n, \alpha)\}$ is a linearly independent subset of $H_{\alpha}\left(\Sigma_{p^{n}}\right)$.

Proof. If $n=0$, the only nonempty $A(p, q, 0, \alpha)$ is $A(p, q, 0,0)=\{(\quad)\}$ for any $q$. In this case define $b(()) \in H_{0}\left(\Sigma_{1}\right)$ as a generator for this one 
dimensional vector space. If $n>0$, let $Q(p, q, n, \alpha)=\left\{\left(j_{1}, j_{2}, \ldots, j_{n}\right) \in\right.$ $\left(\mathbf{Z}^{+}\right)^{n} \mid$ for each $i, j_{i} \equiv 0$ or $-1 \bmod 2(p-1) ; j_{i-1} \leq p j_{i}$ for $i=2, \ldots, n$; $\left.j_{1}>(p-1)\left(j_{2}+\cdots+j_{n}\right) ; j_{1}+j_{2}+\cdots+j_{n}=\alpha ; j_{n}<q(p-1)-1\right\}$. If $\left(j_{1}, \ldots, j_{n}\right) \in Q(p, q, n, \alpha)$, define $a\left(j_{1}, \ldots, j_{n}\right) \in H_{\alpha}\left(\Sigma_{p^{n}}\right)$ as Nakaoka does in $[19$, p. 248].

Nakaoka defines a one-one correspondence

$$
\chi: Q(p, q, n, \alpha) \rightarrow A(p, q, n, \alpha)
$$

by $\chi\left(j_{1}, \ldots, j_{n}\right)=\left(i_{1}, \ldots, i_{n}\right)$ where $i_{k}=q p^{n-k}(p-1)-j_{k}$. Define $b(I)=$ $a\left(\chi^{-1}(I)\right)$; Nakaoka shows that $\{a(J) \mid j \in Q(p, q, n, \alpha)\}$ are linearly independent.

Kahn and Priddy [7, Proposition 3.4] show that $\{b(I) \mid I \in A(p, q, n, \alpha)\}$ is a subset of $\left(\operatorname{im} i_{*}\right)_{\alpha}$. Therefore, we have:

Corollary 14. (Compare with Kahn-Priddy [7, Proposition 3.7].) A vector space basis for $\left(\operatorname{im} i_{*}\right)_{\alpha}$ is $\{b(I) \mid I \in A(p, q, n, \alpha)\}$.

The basis of Corollary 14 will be called the Nakaoka basis for $\widehat{P}_{\alpha, p^{n}}$.

\section{AN ALGEBRA-COALGEBRA OF "UNSTABLE" ADDITIVE COHOMOLOGY OPERATIONS}

There is a "wreath" product on $\hat{H}_{* *}$ (see, e.g., [19 or 7]) which we review here. This product is a function $H_{i}\left(\Sigma_{m}\right) \times H_{j}\left(\Sigma_{n}\right) \rightarrow H_{i+j m}\left(\Sigma_{m n}\right)$.

If $X_{*}$ is a chain complex over $\mathrm{Z} / p$, let $X_{*}^{\otimes m}$ denote the chain complex given by the usual $m$-fold tensor product of $X_{*}^{*}$. The complex $X_{*}^{\otimes m}$ becomes a $\Sigma_{m}$-complex by setting

$$
\sigma\left(x_{1} \otimes \cdots \otimes x_{m}\right)=\varepsilon\left(\sigma, \operatorname{deg} x_{1}, \ldots, \operatorname{deg} x_{m}\right)\left(x_{\sigma^{-1}(1)} \otimes \cdots \otimes x_{\sigma^{-1}(m)}\right)
$$

for $\sigma \in \Sigma_{m}$; here $\varepsilon: \Sigma_{m} \times\left(\mathbf{Z}^{+}\right)^{m} \rightarrow\{ \pm 1\}$ is a function that makes $X_{*}^{\otimes m}$ a $\Sigma_{m}$-complex, see [4] for a precise definition of $\varepsilon$.

Define the wreath product $\left.\Sigma_{m}\right\} \Sigma_{n}$ as the semidirect product of $\Sigma_{m}$ and $\left(\Sigma_{n}\right)^{m}$; where $\Sigma_{m}$ acts on $\left(\Sigma_{n}\right)^{m}$ on the left via permutation of factors. If $M_{*}$ is a chain complex over $\mathbf{Z} / p\left[\Sigma_{n}\right]$ and $L_{*}$ is a chain complex over $\mathbf{Z} / p\left[\Sigma_{m}\right]$, then $L_{*} \otimes M_{*}^{\otimes m}$ becomes a $\left.\mathbf{Z} / p\left[\Sigma_{m}\right\rangle \Sigma_{n}\right]$-complex if we define

$$
\left(\sigma, \tau_{1}, \ldots, \tau_{m}\right)\left(1 \otimes x_{1} \otimes \cdots \otimes x_{m}\right)=\sigma 1 \otimes \sigma\left(\tau_{1} x_{1} \otimes \cdots \otimes \tau_{m} x_{m}\right) .
$$

Proposition 15. If $L_{*}$ and $M_{*}$ are as above, $A$ is a $\mathrm{Z} / p\left[\Sigma_{m}\right]$-module, and $B$ is a $\mathbf{Z} / p\left[\Sigma_{n}\right]$-module, then there is an isomorphism of chain complexes

$$
\varphi:\left(L_{*} \otimes M_{*}^{\otimes m}\right) \otimes_{\Sigma_{m} \Sigma_{n}}\left(A \otimes B^{\otimes m}\right) \rightarrow\left(L_{*} \otimes_{\Sigma_{m}} A\right) \otimes_{\Sigma_{m}}\left(M_{*} \otimes_{\Sigma_{n}} B\right)^{\otimes m} .
$$

Proof. Left to the reader.

Theorem 16 (see, e.g., [4]). If $L_{*}$ is a free acyclic $\Sigma_{m}$-complex and $M_{*}$ is a free acyclic $\Sigma_{n}$-complex, then $L_{*} \otimes M_{*}^{\otimes m}$ is a free acyclic $\left.\Sigma_{n}\right\} \Sigma_{n}$-complex. 
Proposition 15 and Theorem 16 say that we may compute

$$
H_{*}\left(\Sigma_{n} \succ \Sigma_{n}, A \otimes B^{\otimes m}\right)
$$

by computing the homology of the chain complex

$$
\left(L_{*} \otimes_{\Sigma_{m}} A\right) \otimes_{\Sigma_{m}}\left(M_{*} \otimes_{\Sigma_{n}} B\right)^{\otimes m}
$$

where $L_{*}$ and $M_{*}$ are as in Theorem 16. We assume $L_{*}$ and $M_{*}$ are as in Theorem 16 from now on.

If $c \in H_{i}\left(\Sigma_{m}\right)$ and $d \in H_{j}\left(\Sigma_{n}\right)$ define $c \nmid d \in H_{i+j m}\left(\Sigma_{m} \imath \Sigma_{n}\right)$ by $c \nmid d=$ the homology class of

$$
c^{\prime} \otimes\left(d^{\prime} \otimes \underset{d \text { times }}{\cdots} \otimes d^{\prime}\right)
$$

where $c^{\prime} \in L_{i} \otimes_{\Sigma_{m}} \mathbf{Z} / p$ is a cycle representing $c$ and $d^{\prime} \in M_{j} \otimes_{\Sigma_{n}} \mathbf{Z} / p$ is a cycle representing $d$. The reader can check that this definition makes sense.

Now, once and for all, fix embeddings $\rho_{m, n}: \Sigma_{m} \geq \Sigma_{n} \rightarrow \Sigma_{m n}$ for every $m, n \geq 1$. Then if $c, d$ are as above, define $c \circ d \in H_{i+j m}\left(\Sigma_{m n}\right)$ as $\left(\rho_{m, n}\right)_{*}(c<d)$.

Theorem 17 (Nakaoka [19]). If $c \in H_{i}\left(\Sigma_{m}\right)$ and $d \in H_{j}\left(\Sigma_{n}\right)$, and $i$ and $j$ are even if $p$ is odd, then $(c \circ d)^{\wedge}=\hat{c} \circ \hat{d}$.

Proof. (The assumption that $i$ and $j$ are even if $p$ is odd is used because the definition of $\hat{c}$ for $c \in H_{i}\left(\Sigma_{m}\right)$ gives $\hat{c}$ only as an operation on even degree cohomology.) We have

$$
\begin{aligned}
(c \circ d)^{\wedge} & =\left[\left(\rho_{m, n}\right)^{*}(c<d)\right]^{\wedge}=(c<d)^{\wedge}\left(\rho_{m, n}\right) \quad(\text { by }[21]) \\
& =\hat{c} \circ \hat{d} \quad(\text { by Nakaoka [19, Proposition 6.3]). }
\end{aligned}
$$

(We are using the notation of [3].)

From now on, assume $i$ and $j$ are even if $p$ is odd.

Theorem 18. (a) If $\langle 1\rangle$ is the generator of $\widehat{P}_{0,1}$ then $\langle 1\rangle \circ x=x \circ\langle 1\rangle=x$ for every $x \in \widehat{P}_{i, p^{n}}$.

(b) If $x \in \widehat{P}_{i, p^{n}}, y \in \widehat{P}_{j, p^{m}}$ and $z \in \widehat{P}_{k, p^{1}}$ then

(i) $x \circ(y \circ z)=(x \circ y) \circ z$,

(ii) $x \circ(y+z)=x \circ y+x \circ z$, if $j=k$ and $m=l$,

(iii) $(x+y) \circ z=x \circ z+y \circ z$ if $i=j$ and $m=n$.

(c) If $x \in \widehat{P}_{i, p^{n}}$ and $y \in \widehat{P}_{j, p^{m}}$ then $x \circ y \in \widehat{P}_{i+p^{n} j, p^{n+m}}$.

Proof. We use the following basic fact: if $x$ and $y \in H_{\alpha}\left(\Sigma_{\beta}\right)$ then $x=y$ if and only if $\hat{x}_{q}=\hat{y}_{q}$ for some (and hence for all) even $q<\alpha+1$ (see Theorem $4(\mathrm{~b}))$. So, in what follows, $q$ is a sufficiently large even integer.

It is easy to directly compute that $\left(\langle 1\rangle_{q}\right)^{\wedge}=$ identity, so using Theorem 17 , (a) follows. 
By Theorem 17, $\left([x \circ(y+z)]_{q}\right)^{\wedge}=\hat{x}_{q} \circ(\hat{y}+\hat{z})_{q}$. But $\hat{x}_{q}$ is an additive operation, so

$$
\hat{x}_{q} \circ(\hat{y}+\hat{z})_{q}=\hat{x}_{q} \circ \hat{y}_{q}+\hat{x}_{q} \circ \hat{z}_{q}=[(x \circ y+x \circ z)]_{q}^{\wedge} .
$$

The other equalities in (b) actually hold for arbitrary (bihomogeneous) elements of $\widehat{H}_{* *}$, and follow from similar calculations.

By Corollary 5, $\Phi_{\alpha, m}\left(H_{\alpha}\left(\Sigma_{m}\right)\right) \subseteq{ }_{m} H^{m q-\alpha}(\mathbf{Z}, q)$. So

$$
\Phi_{i+p^{n} j, p^{n+m}}(x \circ y) \in_{p^{n+m}} H^{p^{n+m} q-\left(i+p^{n} j\right)}(\mathbf{Z}, q) .
$$

Since $x$ and $y$ correspond to additive operations, so does $x \circ y$. Therefore

$$
\begin{aligned}
\Phi_{i+p^{n} j, p^{n+m}}(x \circ y) & \in_{p^{n+m}} H^{p^{n+m} q-\left(i+p^{n} j\right)}(\mathbf{Z}, q) \\
& \cap A^{p^{n+m} q-\left(i+p^{n} j\right)}(\mathbf{Z}, q, \mathbf{Z} / p) .
\end{aligned}
$$

By Theorem 8, $x \circ y \in \widehat{P}_{i+p^{n} j, p^{n+m}}$. This proves (c).

We conclude that $\widehat{P}_{*, p^{*}}=\left\{\widehat{P}_{i, p^{n}}\right\}_{i \geq 0, n \geq 0}$ (where $i$ is even if $p$ is odd) becomes a bigraded $\mathrm{Z} / p$-algebra with multiplication

$$
\circ: \widehat{P}_{i, p^{n}} \otimes \widehat{P}_{j, p^{m}} \rightarrow \widehat{P}_{i+p^{n} j, p^{n+m}} \text {. }
$$

(Thus this "bigraded algebra" does not satisfy the usual bigrading conventions.)

From now on we assume that $p=2$. There is also a "coalgebra" structure on $\widetilde{P}_{*, 2^{*}}=\left\{\widetilde{P}_{i, 2^{j}}\right\}_{i \geq 0, j \geq 0}$. To discuss this coalgebra structure, we will use the following theorems of Nakaoka and Hung. Let $\Delta_{*, n}: H_{*}\left(\Sigma_{2^{n}}\right) \rightarrow H_{*}\left(\Sigma_{2^{n}}\right) \otimes$ $H_{*}\left(\Sigma^{2^{n}}\right)$ be the homomorphism induced by the diagonal $\Delta: \Sigma_{2^{n}} \rightarrow \Sigma_{2^{n}} \times \Sigma_{2^{n}}$.

Theorem 19 (Nakaoka [20]). Let $a \in H_{i}\left(\Sigma_{2^{n}}\right)$ and suppose that $\Delta_{i, n}(a)=$ $\sum a^{\prime} \otimes a^{\prime \prime}$. Then, for any space $X$ and any $u, v \in H^{q}(X)$, we have $\hat{a}(u \cup v)=$ $\sum\left(a^{\prime}\right)^{\wedge}(u) \cup\left(a^{\prime \prime}\right)^{\wedge}(v)$.

Theorem 20 (Hung [5]). Let $\left\{D_{R} \mid R \in\left(\mathbf{Z}^{+}\right)^{n}, n \geq 0\right\}$ be the Dickson basis of $\widetilde{P}_{*, 2^{n}}$. Then $\Delta_{i, n}\left(D_{R}\right)=\sum_{S+T=R} D_{S} \otimes D_{T}$; where $R=\left(r_{0}, \ldots, r_{n-1}\right) \in\left(\mathbf{Z}^{+}\right)^{n}$ is such that $\sum_{j=0}^{n-1} r_{j}\left(2^{n}-2^{j}\right)=i$.

Corollary 21. For $i \geq 0$ and $n \geq 0, \Delta_{i, n}\left(\widetilde{P}_{i, 2^{n}}\right) \subseteq \bigoplus_{k+1=i} \widetilde{P}_{k, 2^{n}} \otimes \widetilde{P}_{1,2^{n}}$.

Define an operation

$$
\begin{aligned}
\circ \circ: & \left(\bigoplus_{k+1=i} \widetilde{P}_{k, 2^{n}} \otimes \widetilde{P}_{1,2^{n}}\right) \otimes\left(\bigoplus_{r+s=j} \widetilde{P}_{r, 2^{m}} \otimes \widetilde{P}_{s, 2^{m}}\right) \\
& \rightarrow \bigoplus_{t+u=i+2^{n} j} \widetilde{P}_{t, 2^{n+m}} \otimes \widetilde{P}_{u, 2^{n+m}}
\end{aligned}
$$

by $\left(z_{1} \otimes w_{1}\right) \circ \circ\left(z_{2} \otimes w_{2}\right)=\left(z_{1} \circ z_{2}\right) \otimes\left(w_{1} \circ w_{2}\right)$ if $z_{1}, z_{2}, w_{1}$ and $w_{2}$ are bihomogeneous elements of $\widetilde{P}_{*, 2^{*}}$. Let $\bigoplus_{k+1=i} \widetilde{P}_{k, 2^{m}} \otimes \widetilde{P}_{1,2^{m}}$ act on $H^{*}(X) \otimes$ $H^{*}(X)$ (let $X$ be any nice enough space from now on) by the rule

$$
(\alpha \otimes \beta)^{\wedge}(x \otimes y)=\hat{\alpha}(x) \otimes \hat{\beta}(y) .
$$


Lemma 22. $\left[\Delta_{*, *}\left(\theta_{1}\right)\right]^{\wedge} \circ\left[\Delta_{*, *}\left(\theta_{2}\right)\right]^{\wedge}=\left[\Delta_{*, *}\left(\theta_{1}\right) \circ \circ \Delta_{*, *}\left(\theta_{2}\right)\right]^{\wedge}$ as operations on $H^{*}(X) \otimes H^{*}(X) \cong H^{*}(X \times X)$.

Theorem 23. The homomorphisms $\Delta_{i, m}: \widetilde{P}_{i, 2^{m}} \rightarrow \bigoplus_{k+1=i} \widetilde{P}_{k, 2^{m}} \otimes \widetilde{P}_{1,2^{m}}$ are such that $\Delta_{*, *}(x \circ y)=\Delta_{*, *}(x) \circ \circ \Delta_{*, *}(y)$ for each bihomogeneous pair $x, y \in \widetilde{P}_{*, 2^{*}}$. Proof. We mimic Milnor's proof of the existence of the coalgebra structure on the mod-2 Steenrod algebra [15].

Let $c: H^{*}(X) \otimes H^{*}(X) \rightarrow H^{*}(X)$ denote cup product. Then for each $\theta \in \widetilde{P}_{i, 2^{m}}$ there is a unique element $\Delta_{i, m}(\theta) \in \bigoplus_{k+1=i} \widetilde{P}_{k, 2^{m}} \otimes \widetilde{P}_{1,2^{m}}$ such that $\hat{\theta}(c(x \otimes y))=c\left(\left[\Delta_{i, m}(\theta)\right]^{\wedge}(x \otimes y)\right)$ for every space $X$ and every $x, y \in H^{*}(X)$.

Existence is proved by letting $\Delta_{i, m}(\theta)$ be $\Delta_{i, m}(\theta)$, and applying Theorem 19.

For uniqueness, by Theorem 4, $\Phi_{1,2^{m}}: H_{i}\left(\Sigma_{2^{m}}\right) \rightarrow H^{2^{m} q-i}\left(S P^{\infty} S^{q}\right)$ is injective for $q>i+1$ and $m \geq 0$. Thus,

$$
\widetilde{\Phi}_{i, 2^{m}}: \bigoplus_{k+1=i} \widetilde{P}_{k, 2^{m}} \otimes \widetilde{P}_{1,2^{m}} \rightarrow H^{2^{m}(2 q)-i}\left(S P^{\infty} S^{q} \times S P^{\infty} S^{q}\right)
$$

given by $\alpha \otimes \beta \mapsto \Phi_{k, 2^{m(n)}} \otimes \Phi_{1,2^{m(\beta)}}$ is injective if $q>i+1$ and $m \geq 0$. Suppose $\rho_{1}, \rho_{2} \in \bigoplus_{k+1=i} \widetilde{P}_{k, 2^{m}} \otimes \widetilde{P}_{1,2^{m}}$ and $\theta \in \widetilde{P}_{i, 2^{m}}$ are such that

$$
\hat{\theta}(c(x \otimes y))=c\left(\hat{\rho}_{j}(x \otimes y)\right)
$$

for $j=1,2$ and any $x, y$ in any $H^{*}(X)$. Letting $X=S P^{\infty} S^{q} \times S P^{\infty} S^{q}$, $x=l_{\infty} \times 1$ and $y=1 \times l_{\infty}$, we have $c \hat{\rho}_{j}(x \otimes y)=\widetilde{\Phi}_{i, 2^{m}}\left(\rho_{j}\right)$ for $j=1,2$ (by naturality of the operations induced by elements of $\widetilde{P}_{*, 2^{*}}$, and standard properties of the $\times$ and $U$ products). So, $\rho_{1}=\rho_{2}$.

Now, since

$$
\begin{aligned}
& {\left[\theta_{1} \circ \theta_{2}\right]^{\wedge}(c(x \otimes y))=\hat{\theta}_{1}\left(\hat{\theta}_{2}(c(x \otimes y))\right)} \\
& \quad=c\left(\left[\left[\Delta_{*, *}\left(\theta_{1}\right)\right]^{\wedge} \circ\left[\Delta_{*, *}\left(\theta_{2}\right)\right]^{\wedge}\right](x \otimes y)\right) \\
& \quad=c\left(\left[\Delta_{*, *}\left(\theta_{1}\right) \circ \circ \Delta_{*, *}\left(\theta_{2}\right)\right]^{\wedge}(x \otimes y)\right),
\end{aligned}
$$

we have $\Delta_{*, *}\left(\theta_{1} \circ \theta_{2}\right)=\Delta_{*, *}\left(\theta_{1}\right) \circ \circ \Delta_{*, *}\left(\theta_{2}\right)$.

4. A computation in the algebra $\widetilde{P}_{*, p^{*}}$.

Let

$$
D_{n}=\left\{\begin{array}{l}
D_{(1,0 \ldots .0)} \in H_{2^{n}-1}\left(\Sigma_{2^{n}}\right) \quad \text { if } p=2, \\
D_{(\varnothing, 1,0 \ldots .0} \in H_{2\left(p^{n}-1\right)}\left(\Sigma_{p^{n}}\right) \quad \text { if } p>2 ;
\end{array}\right.
$$

$D_{n}$ is an element of the Dickson basis for $\widetilde{P}_{*, p^{*}}$ for each $n \geq 1$. Define $D_{0}$ as $\langle 1\rangle \in H_{0}\left(\Sigma_{1}\right)$. If $\left(r_{1}, \ldots, r_{n}\right) \in\left(\mathbf{Z}^{+}\right)^{n}$, let $\left(\xi_{1}^{r_{1}} \xi_{2}^{r_{2}} \ldots \xi_{n}^{r_{n}}\right)^{\text {dual }}$ denote the element of the Steenrod algebra $\mathscr{A}_{p}$ dual to the monomial $\xi_{1}^{r_{1}} \ldots \xi_{n}^{r_{n}}$ in the Milnor basis of $\mathscr{A}_{p}^{*}$, with respect to that basis. 
The following theorem is a special case of theorems of Mui [17, 18]:

Theorem 24 (Mui). (a) If $p=2$,

$$
\left(\widehat{D}_{n}\right)_{q}=\left\{\begin{array}{l}
0 \quad \text { if } q<1, \\
\left(\xi_{n}^{q-1}\right)^{\text {dual }} \text { if } q \geq 1 .
\end{array}\right.
$$

(b) If $p>2$, and $q$ is even,

$$
\left(\widehat{D}_{n}\right)_{q}=\left\{\begin{array}{l}
0 \quad \text { if } q<2 \\
\left(\xi_{n}^{(q-2) / 2}\right)^{\text {dual }}
\end{array} \text { if } q \geq 2 .\right.
$$

Therefore,

$$
\left(D_{n} \circ D_{m}\right)_{q}^{\wedge}=\left\{\begin{array}{r}
\left(\widehat{D}_{n}\right)_{2^{m}(q-1)+1} \circ\left(\widehat{D}_{m}\right)_{q}=\left(\xi_{n}^{2^{m}(q-1)}\right)^{\text {dual }} \circ\left(\xi_{m}^{q-1}\right)^{\text {dual }} \\
\quad \text { if } p=2, q \geq 1 \\
\left(\widehat{D}_{n}\right)_{p^{m} q-\left(2 p^{m}-2\right)} \circ\left(\widehat{D}_{m}\right)_{q}=\left(\xi_{n}^{\left(p^{m}(q-2) / 2\right)}\right)^{\text {dual }} \circ\left(\xi_{m}^{(q-2) / 2}\right)^{\text {dual }} \\
\quad \text { if } p>2, q \text { is even, } q \geq 2 .
\end{array}\right.
$$

In particular, if $p=2,\left(D_{n} \circ D_{m}\right)_{1}^{\wedge}=$ identity; and if $p$ is odd, $\left(D_{n} \circ D_{m}\right)_{2}^{\wedge}=$ identity. So, $D_{n} \circ D_{m} \neq 0$. But we see that

$$
D_{n} \circ D_{m} \in \begin{cases}\widetilde{P}_{2^{n+m}-1,2^{n+m}} & \text { if } p=2, \\ \widetilde{P}_{2\left(p^{n+m}-1\right), p^{n+m}} & \text { if } p>2 ;\end{cases}
$$

and by inspecting the Dickson basis for $\widetilde{P}_{*, p^{*}}$, that $\left\{D_{n+m}\right\}$ is a basis for

$$
\begin{cases}\widetilde{P}_{2^{n+m}-1,2^{n+m}} & \text { if } p=2, \\ \widetilde{P}_{2\left(p^{n+m}-1\right), p^{n+m}} & \text { if } p>2 .\end{cases}
$$

In any case, $D_{n} \circ D_{m}$ is a nonzero scalar multiple of $D_{n+m}$. Again, comparing the cohomology operations in degree 1 (or 2 , if $p>2$ ) implies that $D_{n} \circ D_{m}=$ $D_{n+m}$.

On the other hand, Milnor's formula [15] for computing $\left(\xi_{k}^{i}\right)^{\text {dual }} \circ\left(\xi_{1}^{j}\right)^{\text {dual }}$ says that $(\alpha=q-1$, if $p=2 ; \alpha=(q-2) / 2$, if $p>2)$

$$
\left(\xi_{n}^{p^{m} \alpha}\right)^{\text {dual }} \circ\left(\xi_{m}^{(x)}\right)^{\text {dual }}=\left\{\begin{array}{c}
\sum_{0 \leq x \leq \alpha}\left(\xi_{n}^{(\alpha-x)\left(p^{n}+1\right)} \xi_{2 n}^{x}\right)^{\text {dual }} \cdot b(x, q, p, n) \\
\text { if } m=n, \text { and } q \text { is even if } p>2, \\
\sum_{0 \leq x \leq \alpha}\left(\xi_{m}^{(\alpha-x) p^{m}} \xi_{n}^{(x-x} \xi_{n+m}^{x}\right) \text { dual } \\
\text { if } m \neq n, \text { and } q \text { is even if } p>2 .
\end{array}\right.
$$

Here, $b(x, q, p, n)$ is a binomial coefficient depending on the indicated parameters. 
So, for example, if $p=2, n \neq m$, and $q \geq 1$, we have a relation

$$
\sum_{0 \leq x<q-1}\left(\xi_{n}^{(q-1-x) 2^{m}} \xi_{m}^{q-1-x} \xi_{n+m}^{x}\right)^{\text {dual }} \equiv 0
$$

in degree $q$.

\section{CONCLUSION}

In summary, this paper points out how the work of others on mod- $p$ cohomology operations and the mod- $p$ cohomology of the symmetric groups can be used to compute a basis for an analogue of the Newton primitives (i.e., the Adams operations) in complex $K$-theory.

Although the additive cohomology operations induced by these primitives are, strictly speaking, unstable, they are essentially stable because Cartan [2] has told us that every additive operation in degree $q$ in mod- $p$ cohomology is given by an appropriate element (depending on $q$ ) of the Steenrod algebra.

One could ask whether or not the same is true of other cohomology theories. In other words, suppose that $\left(h^{*}, h_{*}\right)$ is a cohomology-homology theory with a transfer, a Kunneth formula, an analogue of Steenrod's power map and whatever else is necessary so that it makes sense to discuss primitives in $\bigoplus_{n \geq 0} h_{*}\left(B \Sigma_{n}\right)$ and the cohomology operations they induce. Do you get any essentially unstable additive operations on $h^{*}$ in this way?

\section{BIBLIOGRAPHY}

1. M. F. Atiyah, Power operations in K-theory, Quart. J. Math. (2) 17 (1966), 165-193.

2. H. Cartan, Séminaire H. Cartan 1954-1955, Paris.

3. J. Duflot, A Hopf algebra associated to the cohomology of the symmetric groups, Proc. Sympos. Pure Math., vol. 47, Amer. Math. Soc., Providence, R.I., 1987, pp. 171-186.

4. L. Evens, The cohomology ring of a finite group, Trans. Amer. Math. Soc. 101 (1961), 224-239.

5. N. H. V. Hung, The mod-2 cohomology algebras of symmetric groups, Japan J. Math. 13 (1987), 169-208.

6. - The mod-p cohomology algebras of symmetric groups, preprint.

7. D. S. Kahn and S. B. Priddy, On the transfer in the homology of the symmetric groups, Math. Proc. Cambridge Philos. Soc. 83 (1978), 91-101.

8. A. Liulevicius, Arrow's, symmetries and representatiosn rings, J. Pure Appl. Algebra 19 (1980), 259-273.

9. I. Madsen, On the action of the Dyer-Lashof algebra in $H_{*}(G)$, Pacific J. Math. 60 (1975), 235-275.

10. I. Madsen and R. J. Milgram, The classifying spaces for surgery and cobordism of manifolds, Ann. of Math. Studies, no. 92, Princeton Univ. Press, 1979.

11. B. M. Mann, The cohomology of the symmetric groups, Trans. Amer. Math. Soc. 242 (1978), 157-183.

12. J. P. May, The homology of $E_{\infty}$ spaces, Lecture Notes in Math., vol. 533, Springer-Verlag. 1976, pp. 1-68.

13. J. McClure, Power operations in $H^{d}$-ring theories, Lecture Notes in Math., vol. 1176, SpringerVerlag. 1986, pp. 249-290. 
14. J. Milnor, The Steenrod algebra and its dual, Ann. of Math. 67 (1958), 150-171.

15. J. Milnor and J. C. Moore, On the structure of Hopf algebras, Ann. of Math. 81 (1965), $211-$ 264.

16. H. Mui, Modular invariant theory and cohomology algebras of symmetric groups, J. Fac. Sci. Univ. Tokyo 22 (1975), 319-369.

17. _ Cohomology operations derived from the modular invariants, preprint.

18. __ Dickson invariants and the Milnor basis of the Steenrod algebra, preprint.

19. M. Nakaoka, Homology of the infinite symmetric group, Ann. of Math. 73 (1981), 228-257.

20. __ Note on cohomology algebras of symmetric groups, J. Math. Osaka City Univ. 13 (1962), 45-55.

21. N. E. Steenrod, Homology groups of symmetric groups and reduced power operations, Proc. Nat. Acad. Sci. U.S.A. 39 (1953), 213-223.

Department of Mathematics, Colorado State University, Fort Collins, Colorado 80523 